\title{
P-201
}

\section{Aerosolized Honey as a Regenerative Agent in Animal Model of Asthma}

\author{
Kamaruzaman $\mathrm{NA}^{1}$, Sulaiman $\mathrm{SA}^{2}$, Gurjeet $\mathrm{K}^{3}$ and Yahaya $\mathrm{BH}^{1}$ \\ ${ }^{I}$ Cluster of Regenerative Medicine, Advanced Medical \& Dental Institute, Universiti Sains Malaysia, 13200 Kepala \\ Batas, Penang, Malaysia; ${ }^{2}$ Department of Pharmacology, School of Medical Sciences, Universiti Sains Malaysia, 16150 \\ Kubang Kerian, Kelantan, Malaysia ${ }^{3}$ Institute for Research in Molecular Medicine, Universiti Sains Malaysia, 11800 , \\ Penang, Malaysia
}

Asthma is a disease characterized by infiltration of inflammatory cells into lung tissue, excessive mucus production, chronic airway obstruction and airway hyperresponsiveness (1). Inhaled corticosteroid is a common therapy for asthma. However, prolonged usage of drug therapy may lead to several side effects (2). Honey has been extensively studied and claimed to be effective in treating cough and asthma. Honey nebulisation has been proven effective in treating upper respiratory disease (3). However, the effects of aerosolized honey in reducing asthma-related symptoms at the cellular level have not been studied. The aim of this study was to determine the effects of honey on histopathology of airways in rabbits induced to have chronic lung disease that mimicked the human condition of asthma. The effects of honey to act as both rescue and preventive agents in alleviating asthmatic symptoms were tested. In both rescue and preventive treatment groups, 12 New Zealand white rabbits $(\mathrm{n}=3)$ were sensitized via intraperitoneal (ip) injections of $0.1 \mathrm{mg} / \mathrm{ml}$ ovalbumin (OVA) with $10 \mathrm{mg} / \mathrm{ml}$ aluminium hydroxide (alum) at day 1 and day 14. Sterile phosphate buffer saline (PBS) was used to act as negative control. Following the ip injections for OVA-treated group ( $\mathrm{n}=3$ ) (positive control group), rabbits were exposed to OVA inhalation (10mg/ml) for 3 subsequent days starting from day 28 and euthanized at day 31. In negative control group, PBS was given in a similar manner as OVA-treated group. For rescue treatment group $(n=3)$, following the ip injections, at day 23 until day 27, animals were treated with $25 \%$ of honey using aerosolisation technique followed by euthanasia at day 28 . For preventive treatment group $(\mathrm{n}=3)$, following second ip injection with OVA, aerosolized honey was continuously given from day 23 until day 27. Subsequently, rabbits were further exposed to OVA inhalation for 3 days starting from day 28 until day 30 and euthanized on day 31 . For each treatment, $2 \mathrm{ml}$ of honey was aerosolised for $20 \mathrm{~min}$ duration. Histopathological changes of the airways were determined using H\&E and AB-PAS staining. A massive infiltration of eosinophils, lymphocytes and mononuclear cells were observed in OVA-treated animals. The airways also showed disruption of epithelium, goblet cell hyperplasia and thickened subepithelial smooth muscle layer, which are characteristic of asthma. Animals exposed to aerosolised honey, especially in the rescue group, showed reduction of these inflammatory changes. In conclusion, this study shows that aerosolised honey are effective in reducing asthma-related cellular changes in models of rabbit-induced chronic lung disease.

\section{ACKNOWLEDGEMENT}

ScienceFund Grant 305/CIPPT/613224, Ministry of Science, Technology \& Innovation (MOSTI), Universiti Sains Malaysia

\section{REFERENCES}

[1] Eun JY, Lee JS, Yun CY, Kim JH, Kim JS, Kim DH, Kim IS. Inhibitory effects of Duchesnea chrysantha extract on ovalbumin-induced lung inflammation in a mouse model of asthma. J Ethnopharmacol 2008; 118: 102-7

[2] Balaha MF, Tanaka H, Yamashita H, Rahman MNA, Inagaki N. Oral Nigella sativa oil ameliorates ovalbumin-induced bronchial asthma in mice. Intern Immunopharmacol 2012; 14: 224-31.

[3] Maksoud AM, Rahman AM. Bee honey nebulisation as a non traditional treatment of acute bronchial asthma in infants and children. First North-South Conference and Workshops on Pharmacogenetics (Beating the Gene:From the Bench to the Bedside) 2006; p. 115. 